\title{
Two Cases of Gouty Sacroiliitis Evaluated by Dual-energy Computed Tomography
}

TOMOKA AO, MD, Department of Allergy and Rheumatology, Graduate School of Medicine, University of Tokyo; HIROFUMI SHODA, MD, PhD, Department of Allergy and Rheumatology, Graduate School of Medicine, University of Tokyo; KAZUHIKO YAMAMOTO, MD, PhD, Department of Allergy and Rheumatology, Graduate School of Medicine, University of Tokyo, Tokyo, Japan. Address correspondence to Dr. H. Shoda, 7-3-1 Hongo, Bunkyo-ku, Tokyo, Japan. E-mail: shoda-tky@umin.ac.jp. J Rheumatol 2016;43:1146-7; doi:10.3899/jrheum.151454

Dual-energy computed tomography (DECT) is a method to detect urate crystals in gouty joints. DECT uses 2 separate radiography tubes producing differing energy levels, which allows an identification of urate crystals in articular and periarticular locations ${ }^{1}$. The usefulness of DECT has been reported for a diagnosis of gouty spondyloarthritis ${ }^{2,3}$. We report 2 cases of gouty sacroiliitis evaluated by DECT.

Case 1. A 44-year-old man was admitted because of multiple peripheral joint swelling, tophus, and tenderness in his sacroiliac joints (SIJ). He had a 14-year history of gout, and an 8-year history of inflammatory back pain. Laboratory tests showed elevated C-reactive protein (CRP; $14.26 \mathrm{mg} / \mathrm{dl}$ ) and hyperuricemia $(9.9 \mathrm{mg} / \mathrm{dl})$. His HLA-B27 was positive. A joint fluid aspiration from ankles contained urate crystals. Magnetic resonance imaging (MRI) showed bone erosion, subchondral osteomyelitis, and synovitis in bilateral SIJ. DECT demonstrated urate crystals attached to SIJ (Figure 1A and $1 \mathrm{~B})$.

Case 2. A 40-year-old woman with a 10-year history of untreated hyperuricemia was admitted because of peripheral arthritis and tophus. She also claimed low back pain. Laboratory tests showed elevated CRP $(39.9 \mathrm{mg} / \mathrm{dl})$ and hyperuricemia $(8.2 \mathrm{mg} / \mathrm{dl})$. HLA-B27 was negative. A joint fluid aspiration from elbows contained urate crystals and
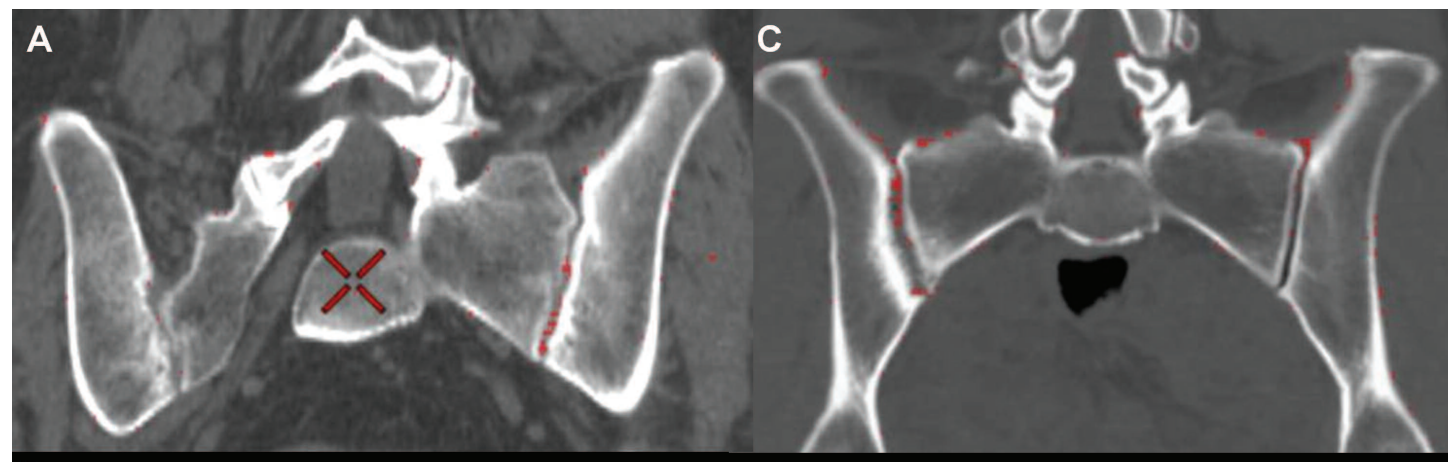

B

D

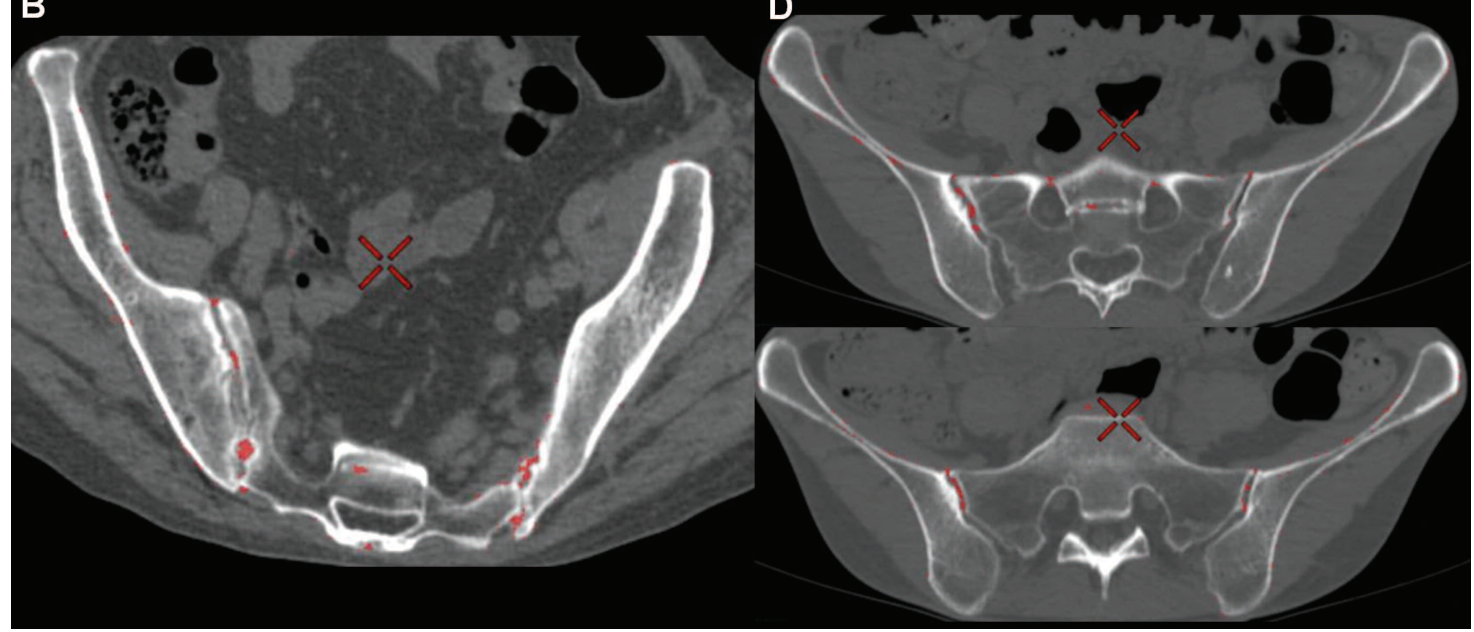

Figure 1. DECT of sacroiliac joints in gouty sacroiliitis. DECT was performed by GE Discovery CT750HD in accordance with manufacturer's protocol. Red spots indicate monosodium urate crystal deposits. (A and B) Case 1. (C and D) Case 2. DECT: dual-energy computed tomography. 
neutrophils. Sacroiliitis was suspected by sclerosis in computed tomography and synovitis in MRI. DECT showed urate crystals attached to the right SIJ (Figure 1C and 1D). Both cases were treated by steroid and febuxostat; however, they showed recurrences of gouty attacks.

In this way, DECT provides important evidence for a diagnosis of gouty spondyloarthritis with minimal invasion.

\section{ACKNOWLEDGMENT}

We thank Dr. Yusuke Sugimori, Dr. Oh Sasaki, Dr. Hiroaki Harada, and Dr. Keishi Fujio from the Department of Allergy and Rheumatology for their diagnosis and assistance with this report.

\section{REFERENCES}

1. Nicolaou S, Yong-Hing CJ, Galea-Soler S, Hou DJ, Louis L, Munk P. Dual-energy CT as a potential new diagnostic tool in the management of gout in the acute setting. AJR Am J Roentgenol 2010;194:1072-8

2. Dhaese S, Stryckers M, Van Der Meersch H, Terryn W, Van Laecke S. Gouty arthritis of the spine in a renal transplant patient: a clinical case report: an unusual presentation of a common disorder. Medicine 2015;94:e676.

3. Parikh P, Butendieck R, Kransdorf M, Calamia K. Detection of lumbar facet joint gouty arthritis using dual-energy computed tomography. J Rheumatol 2010;37:2190-1. 